\title{
Single Photon Emission Computed Tomography Using 99mTc-HM-PAO in the Routine Evaluation of Alzheimer's Disease
}

\author{
T.A. Hurwitz, W. Ammann, D. Chu, C. Clark, J. Holden and R. Brownstone
}

\begin{abstract}
Regional cerebral blood flow was studied in 7 patients with clinically suspected Alzheimer's disease and 10 normal controls by single photon computed emission tomography (SPECT) using HM-PAO. All patients with Alzheimer's disease and no controls had parietal lobe hypoperfusion which was usually bilateral. In patients with more severe dementia hypoperfusion extended into the frontal lobes. Parietal lobe hypoperfusion corresponds to parietal lobe degeneration which is the one of the first neocortical regions to show the typical degenerative changes of Alzheimer's disease. SPECT with HM-PAO is a non-invasive investigation available in most nuclear medicine departments and complements existing tests in the routine evaluation of patients presenting with dementia.
\end{abstract}

\begin{abstract}
RÉSUMÉ: Tomographie par émission de photon unique utilisant le $99 \mathrm{mTc}-H M-P A O$ dans l'évaluation de routine de la maladie d'Alzheimer. Nous avons étudié le flot sanguin régional cérébral chez 7 patients avec maladie d'Alzheimer suspectée en clinique et 10 contrôles normaux par tomographie par émission de photon unique (SPECT) utilisant le HM-PAO. Tous les patients atteints de la maladie d'Alzheimer et aucun des contrôles avaient une hypoperfusion du lobe pariétal qui était habituellement bilatérale. Chez les patients qui avaient une démence plus sévère, l'hypoperfusion s'étendait aux lobes frontaux. L'hypoperfusion du lobe pariétal correspond à la dégénérescence du lobe pariétal qui est une des premières régions du néocortex à manifester les changements dégénératifs typiques de la maladie d'Alzheimer. Le SPECT avec HM-PAO est une technique d'investigation non-invasive disponible dans la plupart des départements de médecine nucléaire et complémente les épreuves actuelles dans l'évaluation de routine des patients qui présentent une démence.
\end{abstract}

Can. J. Neurol. Sci. 1991; $18: 59-62$

Alzheimer's disease can only be conclusively diagnosed by cerebral biopsy or at post mortem. Histology shows neuronal loss, neurofibrillary tangles, senile plaques, loss of cortical lamination, gliosis and spongiosis. These degenerative changes do not involve the brain diffusely but have a characteristic regional distribution which has only recently been fully appreciated. The medial limbic temporobasal areas are the most severely affected. In the neocortex the posterior cingulate gyrus and the superior parietal lobule are the first to show the typical degenerative changes followed by the inferior and middle temporal gyrus and inferior parietal lobule and thereafter by the frontal and occipital lobes and superior temporal gyrus. The anterior cingulate gyrus, the sensorimotor cortex and the calcarine cortex are spared until late in the disease. 1
As non-invasive diagnostic aids, neuroimaging techniques such as cranial computed tomography (CT) and magnetic resonance imaging (MRI) have limited value and usually show generalized atrophy which correlates poorly with mild to moderate dementia. ${ }^{2}$ Positron emission tomography (PET) using fluorodeoxyglucose (FDG) to measure regional cerebral glucose metabolism has proved to be much more useful. Hypometabolic cortical areas have been shown by several groups of investigators. ${ }^{2-5}$ Symmetric or asymmetric hypometabolism is present in the parietal lobes in early Alzheimer's disease which extends to the temporal and frontal lobes as the disease progresses. PET, however, is an expensive and scarce resource and is not available for routine clinical use.

From the Departments of Psychiatry (TAH, CC), Nuclear Medicine (WA, DC) and Pathology (JH), University of British Columbia, and the Department of Physiology (RB), University of Manitoba

Presented at the 38th Annual Meeting of the Canadian Psychiatric Association, Halifax, N.S., 28th - 30th September 1988

Received June 6, 1990. Accepted in final form October 22, 1990

Reprint requests to: Dr. T.A. Hurwitz, Department of Psychiatry, University Hospital, UBC Site, 2255 Wesbrook Mall, Vancouver, B.C., Canada V6T $2 \mathrm{Al}$ 
Regional cerebral blood flow which is closely coupled to regional cerebral glucose metabolism ${ }^{6.7}$ shows a similar pattern of hypoperfusion and may be studied by single photon emission computed tomography (SPECT) using the rotating gamma camera which is available in most nuclear medicine departments. Several radiopharmaceuticals are available for this purpose. Recently 99mTc-hexamethylpropylene amineoxime (HM-PAO) has become available for routine clinical use in Canada. HMPAO behaves like a chemical microsphere ${ }^{8}$ and gives images of regional cerebral perfusion. We performed SPECT using HMPAO on 7 patients with Alzheimer's disease to determine its value in the routine evaluation of patients presenting with dementia.

\section{MeThod}

Seven patients who met DSM-III-R ${ }^{9}$ criteria for Alzheimer's disease (Primary Degenerative Dementia of the Alzheimer Type) were studied. There were 4 females and 3 males with a mean age of 65 years (range 49-76) (Table 1). All presented with a 1-3 year progressive impairment of memory and other intellectual functions. All patients underwent detailed physical and neurological examinations and had a computed tomographic brain scan. Thyroid hormone levels, vitamin B 12, serum folate and serology for syphilis were obtained for each patient to exclude other diseases that may cause dementia. The Mini Mental State (MMS) ${ }^{10}$ was administered and the severity of dementia determined by the Clinical Dementia Rating (CDR) 11 which divides patients into questionable (0.5), mild (1), moderate (2) or severe (3) groups. All patients or their family or guardian consented to SPECT with HM-PAO which is approved for routine clinical use in the University Hospital, University of British Columbia. Table 1 shows demographic data and salient clinical and radiographic features. Four patients were classified as questionable or mild, 2 as moderate and 1 as severe dementia by the CDR. One patient (patient 5) died from bronchopneumonia complicating chronic lymphocytic leukemia. Autopsy showed mild brain atrophy with typical changes of Alzheimer's disease. Ten normal volunteers, 8 females and 2 males with a mean age of 68 years (range 56-75) were studied as controls. Each control subject underwent general and neurological examinations and had a MMS evaluation and CDR. All controls were normotensive, and free of a previous history of vascular disease, had normal general and neurological examinations and had MMS scores of 30 and CDR's of 0. Normal controls all gave written informed consent to participate in the SPECT protocol which was approved by the university ethics committee.

Patients and controls were scanned with the Siemens ZLC 3700 orbiter gamma camera system which has a resolution of 20 $\mathrm{mm}$ full width at half maximum in the plane of the reconstructed image. The patient's head was positioned in a moulded plastic headrest so that the inferior orbital rim was perpendicular to the camera face. The head was stabilized with tape. The camera rotated around the head in 64 steps imaging for 30 seconds at each position. A low energy all purpose collimator was used since phantom studies with a high resolution collimator had yielded lower counts with no appreciable increase in resolution. The raw counts were stored in a computer using a $64 \times 64$ pixel matrix. Tomographic slices were reconstructed without attenuation correction to produce a series of 1 pixel $(6.5 \mathrm{~mm})$ thick axial slices. This yielded an average of 35 slices for each brain. Coronal and sagittal images were reconstructed from the axial counts and were displayed separately or simultaneously to give a three dimensional display of the brain at any chosen level.

HM-PAO was supplied as freeze-dried mixture which was reconstituted with $99 \mathrm{~m} \mathrm{Tc}$-pertechnetate solution and a dose of $10-15 \mathrm{mCi}$ was injected intravenously usually within 10 minutes of preparation. During injection the patients lay in a darkened quiet room with a towel placed over the eyes to minimize environmental stimuli. Imaging commenced within 15 to 60 minutes after injection. The reconstructed images were coded, mixed among scans of non Alzheimer's patients and then evaluated by two individuals who were blind to any clinical information. Regional hypoperfusion was identified by agreement on the basis of visual inspection of the displayed images. Only regions of hypoperfusion that were obvious and encompassed at least 20 pixels were recorded as being present. Scans were also analyzed using a semiquantitative method. Regions of interest (ROI) were placed on the parietal, posterior temporal and frontal lobes and cerebellum and the average counts per pixel measured. Two methods were used. In the first, irregular ROIs had a variable pixel size because they were drawn to encompass regions of hypoperfusion, if present, or to encompass the greater part of each specified region. Left and right lobe ROIs for each patient were identical but differed between patients. The cerebellar ROI

\begin{tabular}{|c|c|c|c|c|c|c|c|}
\hline Patient & Age & Sex & $\begin{array}{l}\text { Duration } \\
\text { in Years }\end{array}$ & MMS & CDR & $\begin{array}{l}\text { Neurological } \\
\text { Examination }\end{array}$ & CT \\
\hline 1 & 64 & $\mathrm{~F}$ & 1 & 24 & 0.5 & normal & atrophy \\
\hline 2 & 58 & M & 2 & 21 & 0.5 & normal & atrophy \\
\hline 3 & 64 & M & 3 & 17 & 1 & normal & atrophy \\
\hline 4 & 73 & $\mathrm{~F}$ & 2 & 25 & 1 & normal & normal \\
\hline 5 & 76 & M & 3 & 16 & 2 & $\begin{array}{l}\text { frontal release signs } \\
\text { unsteady gait } \\
\text { incontinent }\end{array}$ & atrophy \\
\hline 6 & 71 & $\mathrm{~F}$ & 3 & 9 & 2 & frontal release signs & atrophy \\
\hline 7 & 49 & $\mathrm{~F}$ & 3 & 0 & 3 & $\begin{array}{l}\text { paratonic rigidity } \\
\text { frontal release signs }\end{array}$ & atrophy \\
\hline
\end{tabular}

MMS - Mini Mental State

CDR - Clinical Dementia Rating 
was drawn to encompass both cerebellar hemispheres. In the second method regular ROIs of 36 pixels were placed over regions of hypoperfusion or specified regions. The cerebellar ROI was obtained by placing the fixed ROI on each lobe and dividing by 2 . For both methods ROI standardization was accomplished by computing a ratio of $\mathrm{ROI} /$ cerebellum. The cerebellum was used to standardize ROIs since the cerebellum is usually spared in Alzheimer's disease.' Differences between patients and controls for each standardized region were analyzed using Bonferonni correction for multiple t-tests. For semiquantitative analysis the number of standardized ROIs from patients that fell below the lowest standardized ROI from controls for each corresponding region was tabulated.

\section{RESULTS}

As a group, patients with Alzheimer's disease showed significantly reduced perfusion in parietal and temporal regions bilaterally when compared to controls with both semiquantitative methods of analysis (Table 2). On visual inspection no control patient had any regions of hypoperfusion. All Alzheimer's patients had hypoperfusion in the left parietal region. Hypoperfusion was present in the right parietal region in 5 Alzheimer's patients. In other regions hypoperfusion was noted in various combinations (Table 3 ) with frontal hypoperfusion occurring in those patients who were more severely demented. (CDR 2 or 3) In controls, for all regions analyzed, no standardization ratio was lower than 0.86 using irregular ROI to cerebellum ratios. Similarly in controls no standardized ratio was lower

\section{Table 2}

\section{Irregular Regions of Interest (ROI)*}

$\mathrm{ROI} /$ Cerebellum Ratio

\begin{tabular}{lccccc} 
& \multicolumn{2}{c}{$\begin{array}{c}\text { normal } \\
\mathbf{n}=\mathbf{1 0}\end{array}$} & \multicolumn{2}{c}{$\begin{array}{c}\text { Alzheimer's } \\
\mathbf{n}=\mathbf{7}\end{array}$} & t-value \\
& mean & sd & mean & sd & \\
\hline Left temporal region & 0.99 & $(0.05)$ & 0.84 & $(0.07)$ & $5.46^{* *}$ \\
Right temporal region & 0.99 & $(0.05)$ & 0.88 & $(0.07)$ & $3.73^{* *}$ \\
Occipital region & 1.00 & $(0.04)$ & 0.98 & $(0.04)$ & 1.49 \\
Left frontal region & 1.01 & $(0.04)$ & 0.98 & $(0.08)$ & 0.98 \\
Right frontal region & 1.02 & $(0.06)$ & 0.98 & $(0.09)$ & 1.20 \\
Left parietal region & 0.95 & $(0.06)$ & 0.78 & $(0.07)$ & $5.86^{* *}$ \\
Right parietal region & 0.95 & $(0.05)$ & 0.86 & $(0.06)$ & $3.39^{* *}$
\end{tabular}

\section{Regular ROI}

ROI/Cerebellum Ratio

\begin{tabular}{lccccc} 
& \multicolumn{2}{c}{$\begin{array}{c}\text { normal } \\
\mathbf{n}=\mathbf{1 0}\end{array}$} & \multicolumn{2}{c}{$\begin{array}{c}\text { Alzheimer's } \\
\mathbf{n}=\mathbf{7}\end{array}$} & t-value \\
& mean & sd & mean & sd & \\
\hline Left temporal region & 0.86 & $(0.05)$ & 0.74 & $(0.09)$ & $3.37^{* *}$ \\
Right temporal region & 0.87 & $(0.04)$ & 0.74 & $(0.08)$ & $4.20^{* *}$ \\
Occipital region & 0.97 & $(0.04)$ & 0.98 & $(0.06)$ & -0.18 \\
Left frontal region & 0.91 & $(\mathbf{0 . 0 6 )}$ & 0.88 & $(0.11)$ & 0.74 \\
Right frontal region & 0.90 & $(0.05)$ & 0.89 & $(0.12)$ & 0.22 \\
Left parietal region & 0.84 & $(0.05)$ & 0.69 & $(0.06)$ & $5.66^{* *}$ \\
Right parietal region & 0.85 & $(0.05)$ & 0.75 & $(0.09)$ & $3.39^{* *}$ \\
\hline
\end{tabular}

* average counts per pixel for regions of interest placed on the cerebellum, parietal, posterior temporal and frontal lobes

** $p \leq 0.05$ using Bonferroni's correction

sd: standard deviation than 0.77 using regular $\mathrm{ROI}$ to cerebellum ratios. Thus $\leq 0.85$ and $\leq 0.76$ respectively were used as the cutoffs to identify regions of hypoperfusion in Alzheimer's patients using either irregular or regular ROI methods of analysis. Using these cutoffs regional hypoperfusion was noted in the left parietal region in all 7 Alzheimer's patients and in the right parietal region in 5 with both semiquantitative methods. For the parietal regions, left and right, visual inspection, irregular ROI and regular ROI methods of analysis yielded identical patterns of hypoperfusion. For other ROIs the different methods of analysis provided overlapping but not identical results. Non agreement was most often in the temporal regions which was less between the visual inspection and irregular ROI ( 2 out of 14 ROIs) than between visual inspection and regular ROI (4 out of 14 ROIs) methods of analysis. In both semiquantitative methods frontal hypoperfusion was also noted in those patients who were more severely demented.

\section{DisCUSSION}

Parietal lobe hypoperfusion was found in all of our patients and was usually bilateral. Two patients had unilateral defects in the left parietal lobe. Asymmetric regions of hypoperfusion in Alzheimer's disease has been previously shown in PET studies using FDG and may correlate with some of the clinical features. ${ }^{3,5}$ Two of our patients (patients 3 and 4) had disproportionate difficulty with language especially word finding and writing. One of these patients (patient 4) had exclusively left sided hypoperfusion in the parietal and temporal lobes on all methods of analysis. Patient 7 who was severely demented and required total nursing care showed bilateral parietal, temporal and frontal lobe hypoperfusion with sparing of the sensorimotor strip, occipital lobe and cerebellum. Patient 6 who was moderately demented was the only other patient who had frontal lobe hypoperfusion. Within the limitations of the small number of patients in this study, our data also lend support to the concept that in Alzheimer's disease, neocortical hypoperfusion commences in the parietal and temporal lobes and extends into frontal lobes as dementia progresses. ${ }^{5}$ If it were possible to image, medial temporal lobe hypoperfusion should be the initial abnormality in Alzheimer's disease. The medial temporal region however lies beneath the resolution of SPECT. In neocortical regions other than the parietal lobes the lack of consistency of regional hypoperfusion between the 3 methods of analysis may be due to early or mild degeneration. This would increase the likelihood of under-identification of regions of hypoperfusion depending upon the sensitivity of each method. When degeneration is

Table 3: Regional Hypoperfusion by Visual Inspection

\begin{tabular}{ccccccccc}
\hline \hline Patient & CDR & \multicolumn{2}{c}{ Parietal } & \multicolumn{2}{c}{ Temporal } & \multicolumn{2}{c}{ Frontal } & Occipital \\
& & left & right & left & right & left right & \\
\hline 1 & 0.5 & + & + & - & - & - & - & - \\
2 & 0.5 & + & + & + & + & - & - & - \\
3 & 1 & + & + & + & - & - & - & - \\
4 & 1 & + & - & + & - & - & - & - \\
5 & 2 & + & - & - & - & - & - & - \\
6 & 2 & + & + & + & - & + & - & - \\
7 & 3 & + & + & + & + & + & + & - \\
\hline
\end{tabular}

regions of hypoperfusion are identified with + 
advanced as in patient 7 all 3 methods of analysis yield identical patterns of hypoperfusion.

Parietal lobe degeneration was detected by all 3 methods of analysis regardless of the degree of dementia and when visualized on functional imaging techniques such as SPECT or PET may be considered the hallmark of Alzheimer's disease. The presence of parietal lobe hypometabolism or hypoperfusion in Alzheimer's disease has been established by previous PET studies using FDG and SPECT studies using Iofetamine I 123.3,4,5,12 Two other SPECT studies using HM-PAO have documented posterior hypoperfusion in patients with Alzheimer's disease. ${ }^{13.14}$ Sixty-seven percent of patients in the study by Neary et al ${ }^{13}$ and $76 \%$ in the study by Gemmell et al ${ }^{14}$ showed posterior hypoperfusion. In both these studies there were no normal controls and the images were assessed by visual analysis only. Perani et al ${ }^{15}$ studied 16 patients with Alzheimer's disease and 16 normal controls and analyzed their data semi-quantitatively using ROI/cerebellar ratios. They found global cerebral hypoperfusion most marked in the frontal and temporoparietal regions.

The failure to find $100 \%$ concordance between the SPECT images and the diagnosis of Alzheimer's disease in previous studies may be due to imaging patients with very early disease or the inclusion of patients with non-Alzheimer dementias since histological confirmation was not obtained in the majority of patients. Parietal lobe hypoperfusion may also not be specific to Alzheimer's disease. Similar patterns of hypoperfusion have been demonstrated in patients with multi-infarct dementia which usually but not always corresponded to infarcts visualized on MR images. ${ }^{14}$ Some of these cases may have represented a combination of Alzheimer's disease and multi-infarct dementia (mixed dementia). Only histological examination can resolve this question.

Thus SPECT images using HM-PAO should not be interpreted in isolation. However the presence of parietal lobe hypoperfusion in a patient presenting with a gradual decline in intellectual functions, a non focal neurological examination and no focal lesions on CT or MRI makes the diagnosis of Alzheimer's disease highly likely. SPECT with HM-PAO is now accessible in most nuclear medicine departments and complements existing tests in the routine evaluation of dementia.

\section{REFERENCES}

1. Brun A, Englund E. Regional pattern of degeneration of Alzheimer's disease: Neuronal loss and histopathological grading. Histopathology 1981; 5: 549-564.

2. McGeer PL, Kamo H, Harrop R, et al. Positron emission tomography in patients with clinically diagnosed Alzheimer's disease. Can Med Assoc J 1986; 134: 597-607.

3. Chase TN, Foster NL, Fedio P, et al. Regional cortical dysfunction in Alzheimer's disease as determined by positron emission tomography. Ann of Neurol 1984; 15: S170-S174.

4. Cutler NR, Haxby JV, Duara R, et al. Brain metabolism as measured with positron emission tomography: Serial assessment in a patient with familial Alzheimer's disease. Neurology 1985; 35: 1556-1561.

5. Duara R, Grady C, Haxby J, et al. Positron emission tomography in Alzheimer's disease. Neurology 1986; 36: 879-887.

6. Baron JC, LeBrun-Grandie PH, Collard PH, et al. Noninvasive measurement of blood flow, oxygen consumption, and glucose utilization in the same brain regions in man by positron emission tomography: Concise communication. J Nucl Med 1982; 23: 391-399.

7. Kuschinsky W, Suda S, Sokoloff L. Local cerebral glucose utilization and blood flow during metabolic acidosis. Am J Physiol 1981; 241: 772-777.

8. Ell PJ, Jarritt PH, Costa DC, et al. Functional imaging of the brain. Semin Nuc Med 1987; 17: 214-229.

9. American Psychiatric Association. Diagnostic and statistical manual of mental disorders (DSM-III-R) 3rd ed - revised. Washington DC: American Psychiatric Association, 1987.

10. Folstein MF, Folstein SE, McHugh PR. "Mini-mental state": A practical method for grading the cognitive state of patients for the clinician. J Psychiatr Res 1975; 12: 189-198.

11. Hughes CP, Berg L, Danziger WL, et al. A new clinical scale for the staging of dementia. Br J Psychiatry 1982; 140: 566-572.

12. Johnston KA, Holman BL, Mueller SP, et al. Single photon emission computed tomography in Alzheimer's disease. Arch Neurol 1988; 45: 392-396.

13. Neary D, Snowden JS, Shields RA, et al. Single photon emission tomography using $99 \mathrm{~m} \mathrm{Tc}-\mathrm{HM}-\mathrm{PAO}$ in the investigation of dementia. J Neurol Neurosurg Psychiatry 1987; 50: 1101-1109.

14. Gemmell HG, Sharp PF, Besson JAO, et al. Differential diagnosis in dementia using the cerebral blood flow agent $99 \mathrm{~m} t \mathrm{c} \mathrm{HM}-\mathrm{PAO}$ : a SPECT study. J Comput Assist Tomogr 1987; 11(3): 398-402.

15. Perani D, Di Piero V, Vallar G, et al. Technetium - 99m HM-PAOSPECT study of regional cerebral perfusion in early Alzheimer's disease. J Nucl Med 1988; 29: 1507-1514. 